Acta Mathematica Sinica, English Series

Sep., 201x, Vol. x, No. x, pp. 1-8

Published online: August 15, 201x

DOI: 0000000000000000

Http://www.ActaMath.com

\title{
Trigonometric series with a generalized monotonicity condition
}

\author{
Lei FENG \\ Institute of Mathematics, Zhejiang Sci-Tech University, Hangzhou 310018 China \\ E-mail:larryleifeng@163.com \\ Vilmos TOTIK \\ MTA-SZTE Analysis and Stochastics Research Group \\ Bolyai Institute, University of Szeged, Szeged, Hungary \\ Department of Mathematics and Statistics, University of South Florida \\ E-mail: totik@mail.usf.edu \\ Songping ZHOU \\ Institute of Mathematics, Zhejiang Sci-Tech University, Hangzhou 310018 China \\ E-mail: songping.zhou@163.com
}

\begin{abstract}
In this paper, we consider numerical and trigonometric series with a very general monotonicity condition. First, a fundamental decomposition is established from which the sufficient parts of many classical results in Fourier analysis can be derived in this general setting. In the second part of the paper a necessary and sufficient condition for the uniform convergence of sine series is proved generalizing a classical theorem of Chaundy and Jolliffe.
\end{abstract}

Keywords uniform convergence, monotonicity, mean value bounded variation, decomposition

MR(2010) Subject Classification 42A20, 42A32

\section{Introduction}

In this paper we consider a generalization of monotonicity for real sequences $\left\{a_{n}\right\}$. The condition we use is that for some $\lambda \geq 2$ and a positive constant $M$ the inequality

$$
\sum_{k=n}^{2 n}\left|\Delta a_{k}\right|:=\sum_{k=n}^{2 n}\left|a_{k}-a_{k+1}\right| \leq \frac{M}{n} \sum_{k=n / \lambda}^{\lambda n}\left|a_{k}\right|
$$

is true for all $n$, where $\sum_{k=n / \lambda}^{\lambda n}$ means $\sum_{n / \lambda \leq k \leq \lambda n}$.

Monotone sequences clearly satisfy (1.1). See the papers [2] [4], [8] [12] for various other variations, of which (1.1) is the most general one. For positive sequences property (1.1) was first introduced in [12], where it was called the Mean Value Bounded Variation (MVBV) condition, and the papers [1], [5] [6], [8] $[10],[12]$ show that (1.1) in the positive case allows one to derive necessary and sufficient conditions for various properties of trigonometric sums in terms of their

Received x x, 201x, accepted x x, 201x

Supported by the European Research Council Advanced Grant (Grant No. 267055) 
coefficient sequences. In the paper [12] it was also shown that from this point of view condition (1.1) cannot be further weakened.

In the present paper we show that in many situations the positivity assumption can be dropped. In particular, for the uniform convergence of sine series condition (1) allows us to derive necessary and sufficient conditions for uniform convergence, thereby obtaining a very general extension of the classical result of Chaundy and Jolliffe.

Throughout the paper, we always use $M$ for the positive constant appearing in (1).

\section{A basic decomposition and sufficient conditions}

The main result of the present section is the following structural theorem which gives a decomposition of any sequence with property (1.1) as a difference of two such nonnegative sequences.

Without loss of generality we may assume $\lambda>8$ and $M>1$ in (1.1). For a sequence $\left\{a_{n}\right\}$ set

$$
b_{n}=\frac{1}{n} \sum_{k=n / \lambda}^{\lambda n}\left|a_{k}\right| .
$$

Theorem 2.1 Let $\left\{a_{n}\right\}$ be an arbitrary sequence with property (1.1) with some $\lambda>8$. Then there is a constant $B$ such that the sequences $\left\{B b_{n}\right\}$ and $\left\{c_{n}=B b_{n}-a_{n}\right\}$ are nonnegative, and they both satisfy (1.1).

Note that this gives the announced decomposition, since $a_{n}=B b_{n}-\left(B b_{n}-a_{n}\right)$.

Actually, we will see that $B=4 M$ is appropriate.

Proof We start with

Lemma 2.2 For all $n$ we have

$$
\left|a_{n}\right| \leq 2 M b_{n} .
$$

Proof Suppose to the contrary that for some $n$ we have $\left|a_{n}\right|>2 M b_{n}$. Then for all $n<k \leq 2 n$ we obtain from property (1.1) for $\left\{a_{n}\right\}$ that

$$
\left|a_{k}\right| \geq\left|a_{n}\right|-\sum_{j=n}^{k-1}\left|\Delta a_{j}\right|>2 M b_{n}-M b_{n}=M b_{n},
$$

so

$$
b_{n} \geq \frac{1}{n} \sum_{k=n}^{2 n}\left|a_{k}\right|>M b_{n}
$$

which is not possible since $M>1$.

Next, we show that $\left\{b_{n}\right\}$ satisfies property (1.1).

Clearly, if (1.1) is true for sufficiently large $n$ then it is true (with a possibly different $M$ ) for all $n$, so in verifying (1.1) we may always assume $n$ to be sufficiently large.

We have, from (2.1),

$$
\begin{aligned}
\left|\Delta b_{k}\right| & =\left|\frac{1}{k} \sum_{j=k / \lambda}^{\lambda k}\right| a_{j}\left|-\frac{1}{k+1} \sum_{j=(k+1) / \lambda}^{\lambda(k+1)}\right| a_{j}|| \\
& \leq \sum_{j=(k+1) / \lambda}^{\lambda k}\left|a_{j}\right| \frac{1}{k(k+1)}+\sum_{k / \lambda \leq j<(k+1) / \lambda} \frac{\left|a_{j}\right|}{k}+\sum_{\lambda k<j \leq \lambda(k+1)} \frac{\left|a_{j}\right|}{k+1} .
\end{aligned}
$$


Therefore,

$$
\begin{aligned}
\sum_{k=n}^{2 n}\left|\Delta b_{k}\right| & \leq \sum_{j=n / \lambda}^{2 \lambda n}\left|a_{j}\right| \sum_{k=j / \lambda}^{\lambda j} \frac{1}{k(k+1)} \\
& +\sum_{j=n / \lambda}^{\lambda(2 n+1)}\left|a_{j}\right|\left(\sum_{\lambda j-1<k \leq \lambda j} \frac{1}{k}+\sum_{j / \lambda-1 \leq k<j / \lambda} \frac{1}{k+1}\right),
\end{aligned}
$$

and this easily gives

$$
\sum_{k=n}^{2 n}\left|\Delta b_{k}\right| \leq \sum_{j=n / \lambda}^{2 \lambda n}\left|a_{j}\right| \frac{\lambda}{j}+\sum_{j=n / \lambda}^{\lambda(2 n+1)}\left|a_{j}\right|\left(\frac{1}{\lambda j-1}+\frac{1}{j / \lambda}\right) \leq \frac{3 \lambda^{2}}{n} \sum_{j=n / \lambda}^{\lambda(2 n+1)}\left|a_{j}\right| .
$$

On the other hand, in

$$
\sum_{k=n / \lambda}^{\lambda n} b_{k}=\sum_{k=n / \lambda}^{\lambda n} \frac{1}{k} \sum_{j=k / \lambda}^{\lambda k}\left|a_{j}\right|
$$

an $\left|a_{j}\right|$ with $n / \lambda \leq j \leq \lambda(2 n+1)$ has coefficient

$$
\sum_{\substack{j / \lambda \leq k \leq \lambda j \\ n / \lambda \leq k \leq n \lambda}} \frac{1}{k}=\sum_{\max (j, n) / \lambda \leq k \leq \lambda \min (j, n)} \frac{1}{k} \geq \frac{1}{\lambda n}(\lambda \min (j, n)-\max (j, n) / \lambda) .
$$

For $n / \lambda \leq j<n$ the right-hand side is

$$
\frac{1}{\lambda n}\left(\lambda j-\frac{n}{\lambda}\right) \geq \frac{1}{\lambda n}\left(n-\frac{n}{\lambda}\right) \geq \frac{1}{2 \lambda},
$$

while for $n \leq j \leq \lambda(2 n+1)$ it is

$$
\frac{1}{\lambda n}\left(\lambda n-\frac{j}{\lambda}\right) \geq \frac{1}{\lambda n}(\lambda n-(2 n+1)) \geq \frac{1}{2} .
$$

Therefore, we obtain from (2.2) that

$$
\sum_{k=n}^{2 n}\left|\Delta b_{k}\right| \leq \frac{6 \lambda^{3}}{n} \sum_{k=n / \lambda}^{\lambda n} b_{k}
$$

which verifies property (1.1) for the sequence $\left\{b_{k}\right\}$.

Finally, we show that $c_{n}:=4 M b_{n}-a_{n}$, which, according to Lemma 2.2, are all nonnegative, also satisfy property (1.1). We follow the preceding proof. Now

$$
\sum_{k=n}^{2 n}\left|\Delta c_{k}\right| \leq 4 M \sum_{k=n}^{2 n}\left|\Delta b_{k}\right|+\sum_{k=n}^{2 n}\left|\Delta a_{k}\right|
$$

and here the last sum is, by property (1.1) for $\left\{a_{n}\right\}$,

$$
\sum_{k=n}^{2 n}\left|\Delta a_{k}\right| \leq M b_{n} \leq \frac{M}{n} \sum_{k=n+1}^{2 n}\left(\left|b_{n}-b_{k}\right|+b_{k}\right) \leq M \sum_{k=n}^{2 n}\left|\Delta b_{k}\right|+\frac{M}{n} \sum_{k=n}^{2 n} b_{k} .
$$

Therefore, in view of (2.3),

$$
\sum_{k=n}^{2 n}\left|\Delta c_{k}\right| \leq 5 M \sum_{k=n}^{2 n}\left|\Delta b_{k}\right|+\frac{M}{n} \sum_{k=n}^{2 n} b_{k} \leq\left(5 M \cdot 6 \lambda^{3}+M\right) \frac{1}{n} \sum_{k=n / \lambda}^{\lambda n} b_{k} .
$$


But, by Lemma 2.2, we have

$$
c_{k} \geq 4 M b_{k}-2 M b_{k} \geq b_{k},
$$

so on the right we can replace $b_{k}$ by $c_{k}$ and we obtain property (1.1) for the sequence $\left\{c_{n}\right\}$.

Corollary 2.3 Suppose that a real sequence $\left\{a_{n}\right\}$ satisfies the condition (1.1), and consider the trigonometric series

$$
S(x) \equiv \sum_{n=1}^{\infty} a_{n} \sin n x
$$

(a) If

$$
\sum_{n=1}^{\infty} \frac{\left|a_{n}\right|}{n}<\infty
$$

then $S$ converges everywhere, and it is the Fourier series of its sum $f(x)$.

(b) If $\lim _{n \rightarrow \infty} n a_{n}=0$, then $S$ converges uniformly.

(c) If, for some $0<\gamma<1$, we have

$$
\sum_{n=1}^{\infty} n^{\gamma-1}\left|a_{n}\right|<\infty
$$

then $x^{-\gamma} f(x)$ is $L^{1}$-integrable.

(d) If $1<p<\infty, 1 / p-1<\gamma<1 / p$ and

$$
\sum_{n=1}^{\infty} n^{p+p \gamma-2}\left|a_{n}\right|^{p}<\infty
$$

then $x^{-\gamma} f(x)$ is $L^{p}$-integrable.

(e) Let $S(x)$ be a Fourier series of an integrable function $f(x) \in L_{2 \pi}$. If $\lim _{n \rightarrow \infty} a_{n} \log n=0$, then $S$ converges to $f$ in $L^{1}$-norm.

Statements (a), (c), (d) and (e) are also true for the cosine series

$$
S(x) \equiv \sum_{n=0}^{\infty} a_{n} \cos n x
$$

except that in (a) the claim is that convergence takes place for all $x \neq 0(\bmod \pi)$. It is easy to see that conditions (2.5) and (2.6) imply (2.4), so the function $f(x)$ in (c) and (d) is well defined.

We note that when $\left\{a_{n}\right\}$ is positive, then the conditions in (b)-(e) are not only sufficient, but also necessary (under the condition (1.1)), e.g. $S$ converges uniformly if and only if $\lim _{n \rightarrow \infty} n a_{n}=$ 0 . When $\left\{a_{n}\right\}$ can change sign, then the necessity of the given conditions may not be always true. However, we shall discuss the uniform convergence case in Section 3, where we shall obtain also the necessity of $n a_{n} \rightarrow 0$.

Proof Corollary 2.3. (a) The claim for nonnegative sequences is in [11]. Therefore, in view of Theorem 2.1, it is enough to show that condition (2.4) implies the same condition for the sequences $\left\{b_{n}\right\}$ and $\left\{c_{n}=B b_{n}-a_{n}\right\}$. Furthermore, in view of Lemma 2.2 we have for $B=4 M$,

$$
2 M b_{n} \leq B b_{n}-2 M b_{n} \leq B b_{n}-a_{n}=c_{n} \leq B b_{n}+2 M b_{n}=6 M b_{n},
$$


so (2.4) needs to be verified only for the sequence $\left\{b_{n}\right\}$. But that is immediate:

$$
\sum_{n} \frac{b_{n}}{n}=\sum_{n} \frac{1}{n^{2}} \sum_{n / \lambda \leq j \leq \lambda n}\left|a_{j}\right|=\sum_{j}\left|a_{j}\right| \sum_{j / \lambda \leq n \leq \lambda j} \frac{1}{n^{2}} \leq \lambda^{3} \sum_{j} \frac{\left|a_{j}\right|}{j}<\infty .
$$

The proof of (b) is similar: the statement for nonnegative sequences is in [12], and we can apply Theorem 2.1, since $a_{n}=o(1 / n)$ implies

$$
b_{n}=\frac{1}{n} \sum_{k=n / \lambda}^{\lambda n} o(1 / k)=o(1 / n),
$$

and the same is true for $\left\{c_{n}\right\}$.

As for (c), the relevant statement for nonnegative sequences was proved in [5] or [7], so, in view of Theorem 2.1, it is sufficient to verify again that (2.5) implies the same for the sequence $\left\{b_{n}\right\}$ (see also $(2.7)$ ), which is immediate:

$$
\sum_{n} n^{\gamma-1} b_{n}=\sum_{n} n^{\gamma-2} \sum_{n / \lambda \leq j \leq \lambda n}\left|a_{j}\right|=\sum_{j}\left|a_{j}\right| \sum_{j / \lambda \leq n \leq \lambda j} n^{\gamma-2} \leq M_{1} \sum_{j} j^{\gamma-1}\left|a_{j}\right|<\infty .
$$

The proof of $(d)$ is similar if we note that the statement for nonnegative sequences is in [1] or [9].

Finally, the verification for (e) is similar to that in (b), and the statement for nonnegative sequences appears in [1] or [10].

As for the relevant results for cosine series, apply Theorem 2.1 in the same fashion, and use the results for nonnegative sequences (see, for example, [11]).

\section{Uniform Convergence: Necessary Condition}

It was proved by Chaundy and Jolliffe (see e.g. [13, Theorem V.1.3]) that if $\left\{a_{n}\right\}$ is a positive decreasing sequence then the series

$$
\sum_{n=1}^{\infty} a_{n} \sin n x
$$

converges uniformly if and only if

$$
\lim _{n \rightarrow \infty} n a_{n}=0 .
$$

There have been many generalizations of this result when the monotonicity of $\left\{a_{n}\right\}$ is replaced by some generalized monotonicity condition, but the positivity of the sequence has usually been assumed. The next theorem gives a very general extension when positivity is not required.

Theorem 3.1 Let a real sequence $\left\{a_{n}\right\}$ satisfy (1.1). Then the series (3.1) converges uniformly if and only if $n a_{n} \rightarrow 0$.

Proof The sufficiency follows from Corollary 2.3, so we only need to prove the necessity. Therefore, assume that the series (3.1) converges uniformly, and we need to show that, under condition (1.1), $n a_{n} \rightarrow 0$. We are actually going to show that

$$
\lim _{n \rightarrow \infty} \sum_{k=n / \lambda}^{\lambda n}\left|a_{k}\right|=0,
$$


and then $n a_{n} \rightarrow 0$ follows from Lemma 2.2.

If condition (1.1) is true for a $\lambda$ then it is true for any larger $\lambda$, therefore we may assume that $\lambda>8$ is an integer.

For an $\varepsilon>0$ choose $N$ so that for $N \leq k \leq l$ we have

$$
\left\|\sum_{j=k}^{l} a_{j} \sin k x\right\|<\varepsilon
$$

Let

$$
B_{n}=\sum_{k=n / \lambda}^{\lambda n}\left|a_{k}\right|
$$

and

$$
B_{n}^{*}=\sum_{k=n / \lambda^{2}}^{\lambda^{2} n}\left|a_{k}\right|
$$

Consider the sets

$$
A_{n}:=\left\{k:\left|a_{k}\right| \geq \frac{B_{n}}{2 \lambda n}, n / \lambda \leq k \leq \lambda n, k \in \mathbb{N}\right\},
$$

and write $\left|A_{n}\right|$ for the number of the elements in $A_{n}$. For each $k \in[n / \lambda, \lambda n]$ we have, in view of Lemma 2.2, the estimate $\left|a_{k}\right| \leq(2 M / k) B_{n}^{*} \leq(2 \lambda M / n) B_{n}^{*}$, hence

$$
B_{n} \leq\left(\sum_{k \in[n / \lambda, \lambda n] \backslash A_{n}} \frac{B_{n}}{2 \lambda n}+\sum_{k \in A_{n}} \frac{2 \lambda M B_{n}^{*}}{n}\right) \leq \frac{\lambda n B_{n}}{2 \lambda n}+\left|A_{n}\right| \frac{2 M \lambda B_{n}^{*}}{n} .
$$

Therefore,

$$
\left|A_{n}\right| \geq n \frac{1}{4 \lambda M} \frac{B_{n}}{B_{n}^{*}}
$$

We select disjoint subsets $S_{1}, \ldots, S_{\kappa_{n}}$ of $[n / \lambda, \lambda n]$ as follows. Set $m_{1}=\min A_{n}$, and select $\nu_{1}$ according to the following procedure:

(i) If for $j=0,1, \cdots, j_{0}, n / \lambda \leq m_{1}+j \leq \lambda n$ the numbers $a_{m_{1}+j}$ have the same sign, and for $j=0,1, \cdots, j_{0}-1,\left|a_{m_{1}+j}\right| \geq B_{n} / 4 \lambda n$ while $\left|a_{m_{1}+j_{0}}\right|<B_{n} / 4 \lambda n$, then let $\nu_{1}=j_{0}$.

(ii) If case (i) is not satisfied for any $j_{0}$, then let $\nu_{1}=k_{0}$ for which $a_{m_{1}+k_{0}}$ is the first element with $m_{1}+k_{0} \in[n / \lambda, \lambda n]$ to become zero or of opposite sign than $a_{m_{1}}$.

(iii) If neither (i) and (ii) happen, then simply let $\nu_{1}=l_{0}$ for which $m_{1}+l_{0}$ is the first number greater than $\lambda n$. Define now

$$
S_{1}=\left\{m_{1}, m_{1}+1, \cdots, m_{1}+\nu_{1}-1\right\} .
$$

Next, set $m_{2}=\min \left(A_{n} \backslash S_{1}\right)$ if this latter set is not empty, and using the same procedure we select $\nu_{2}$ and define

$$
S_{2}=\left\{m_{2}, m_{2}+1, \cdots, m_{2}+\nu_{2}-1\right\} .
$$

We continue this procedure until we reach an $S_{\kappa_{n}}$ for which $A_{n} \backslash\left(S_{1} \cup \cdots \cup S_{\kappa_{n}}\right)=\emptyset$.

Our first task is to give an estimate for $\kappa_{n}$, i.e. for the number of these $S_{j}$ 's. Note first of all that for all $1 \leq j<\kappa_{n}$ we have

$$
\sum_{k \in S_{j}}\left|\Delta a_{k}\right| \geq\left|a_{m_{j}}-a_{m_{j}+\nu_{j}}\right| \geq \frac{B_{n}}{4 \lambda n}
$$


by the choice of the $\nu_{j}$ 's (for $j=\kappa_{n}$ this property may not be true). It is easy to see that (1.1) implies

$$
\sum_{k=n / \lambda}^{\lambda n}\left|\Delta a_{k}\right| \leq \frac{M \lambda^{3}}{n} \sum_{k=n / \lambda^{2}}^{\lambda^{2} n}\left|a_{k}\right|=\frac{M \lambda^{3}}{n} B_{n}^{*}
$$

from which

$$
\frac{M \lambda^{3}}{n} B_{n}^{*} \geq \sum_{k=n / \lambda}^{\lambda n}\left|\Delta a_{k}\right| \geq \sum_{j=1}^{\kappa_{n}-1} \sum_{k \in S_{j}}\left|\Delta a_{k}\right| \geq \sum_{j=1}^{\kappa_{n}-1} \frac{B_{n}}{4 \lambda n}=\left(\kappa_{n}-1\right) \frac{B_{n}}{4 \lambda n},
$$

i.e.

$$
\kappa_{n} \leq 4 M \lambda^{4} \frac{B_{n}^{*}}{B_{n}}+1 \leq 5 M \lambda^{4} \frac{B_{n}^{*}}{B_{n}}
$$

follows.

Note now that all $a_{k}$ for $k \in S_{j}$ are of the same sign, therefore it follows from (3.2) upon substituting $x=\pi /(2 n \lambda)$ and using that for $n / \lambda \leq k \leq \lambda n$ we have

$$
\sin \frac{k \pi}{2 n \lambda} \geq \frac{2}{\pi} \frac{k \pi}{2 n \lambda} \geq \frac{1}{\lambda^{2}}
$$

that

$$
\frac{1}{\lambda^{2}} \sum_{k \in S_{j}}\left|a_{k}\right| \leq\left|\sum_{k \in S_{j}} a_{k} \sin \frac{k \pi}{2 n \lambda}\right|<\varepsilon,
$$

provided $n / \lambda>N$, where $N$ is the threshold for (3.2). On summing up for all $1 \leq j \leq \kappa_{n}$ and using (3.5) it follows that

$$
\sum_{k \in A_{n}}\left|a_{k}\right| \leq \sum_{j=1}^{\kappa_{n}} \sum_{k \in S_{j}}\left|a_{k}\right|<\varepsilon 5 M \lambda^{6} \frac{B_{n}^{*}}{B_{n}}
$$

From here, in view of the definition of the set $A_{n}$ in (3.3) and in view of the bound (3.4), we can infer

$$
\frac{1}{8 \lambda^{2} M} \frac{B_{n}^{2}}{B_{n}^{*}} \leq \varepsilon 5 M \lambda^{6} \frac{B_{n}^{*}}{B_{n}} .
$$

This shows that $B_{n}^{3} /\left(B_{n}^{*}\right)^{2}$ tends to zero as $n \rightarrow \infty$.

Apply this with $n=\lambda^{m}$. Set $q_{m}=B_{\lambda^{m}}, m=1,2, \ldots$. Then $B_{n}^{*} \leq q_{m-1}+q_{m+1}$, hence for these $q_{m}$ we can conclude that

$$
q_{m}^{3} /\left(q_{m-1}+q_{m+1}\right)^{2} \rightarrow 0 \quad \text { as } m \rightarrow \infty .
$$

We show that this implies $q_{m} \rightarrow 0$. Once this is done, the claim $B_{n} \rightarrow 0$ follows, since $B_{n} \leq q_{m}+q_{m+1}$ with $\lambda^{m} \leq n<\lambda^{m+1}$.

To prove $q_{m} \rightarrow 0$ note that (3.6) implies for any $\Lambda>0$ and for some $m \geq m_{\Lambda}$

$$
q_{m-1}+q_{m+1} \geq \Lambda q_{m}^{3 / 2}, \quad m \geq m_{\Lambda} .
$$

Therefore,

$$
2 \limsup _{m \rightarrow \infty} q_{m} \geq \Lambda\left(\limsup _{m \rightarrow \infty} q_{m}\right)^{3 / 2}
$$

Since this is true for any $\Lambda$, we can conclude that this limsup is either 0 (which is what we want to prove) or it is infinity. In the latter case there is an $m \geq m_{3 \lambda}$ for which $q_{m}$ is larger 
than all previous $q_{j}$, and it is larger than 1 . Then (3.7) with $\Lambda=3 \lambda$ gives $q_{m+1} \geq 2 \lambda q_{m}$. In particular, $q_{m+1}$ is larger than any previous $q_{j}$. Now applying again (3.7) (with $m$ replaced by $m+1$ ) we get in the same fashion that $q_{m+2} \geq 2 \lambda q_{m+1} \geq(2 \lambda)^{2} q_{m}$, and so on, in general $q_{m+j} \geq(2 \lambda)^{j} q_{m}>2^{j} \lambda^{j}$ for all $j \geq 1$. However, that is impossible, since (3.2) implies $a_{n} \rightarrow 0$, therefore definitely $q_{m+j} \leq o\left(\lambda^{m+j}\right)$. Hence $\lim \sup q_{m} \rightarrow 0$, and the proof is complete.

\section{References}

[1] Dyachenko M. I., Tikhonov S.: Convergence of trigonometric series with general monotone coefficients. $C$. R. Math. Acad. Sci. Paris, 345:3, 123-126(2007)

[2] Le R. J., Zhou S. P.: A new condition for the uniform convergence of certain trigonometric series. Acta Math. Hungar., 108, 161-169(2005)

[3] Leindler L.: On the uniform convergence and boundedness of a certain class of sine series. Anal. Math., 27, 279-285(2001)

[4] Leindler L.: A new class of numerical sequences and its applications to sine and cosine series. Anal. Math., 28, 279-286(2002)

[5] Tikhonov S.: Trigonometric series with general monotone coefficients. J. Math. Math. Appl., 326, 721$735(2007)$

[6] Tikhonov S.: On $L^{1}$-convergence of Fourier series. J. Math. Math. Appl., 347, 416-427(2008)

[7] Wang M. Z., Zhou S. P.: Applications of MVBV condition in $L^{1}$ integrability. Acta Math. Hungar., 129, 70-80(2010)

[8] Yu D. S., Zhou S. P.: A generalization of the monotonicity condition and applications. Acta Math. Hungar., 115, 247-267(2007)

[9] Yu D. S., Zhou P., Zhou S. P.: On $L^{p}$ integrability and convergence of trigonometric series. Studia Math., 182, 215-226(2007)

[10] Yu D. S., Zhou P., Zhou S. P.: On $L^{1}$-convergence of Fourier series under $M V B V$ condition. Canad. Math. Bull., 52, 627-636(2009)

[11] Zhou S. P.: Monotonicity Condition of Trigonometric Series: Development and Application, Science Press, Beijing, 2012(in Chinese)

[12] Zhou S. P., Zhou P., Yu D. S.: Ultimate generalization to monotonicity for uniform convergence of trigonometric series. Science China Math., 53, 1853-1862(2010)/available: arXiv: math.CA/0611805 v1 27 Nov 2006

[13] Zygmund A.: Trigonometric Series, Cambridge University Press, Cambridge, 1959. 